\title{
Flexible Ferroelectric Organic Crystals
}

\author{
M. Owczarek ${ }^{1}$, K. A. Hujsak ${ }^{2}$, D. P. Ferris ${ }^{1}$, A. Prokofjevs ${ }^{1}$, I. Majerz ${ }^{3}$, P. Szklarz ${ }^{4}$, H. Zhang ${ }^{1}$,

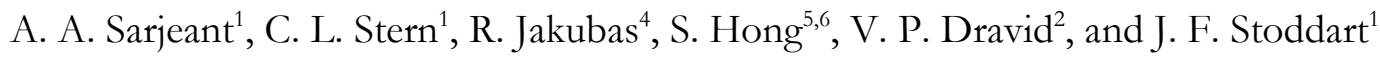

${ }^{1}$ Department of Chemistry, Northwestern University, Evanston, Illinois 60208, USA. 2Department of Materials Science and Engineering, Northwestern University, Evanston, Illinois 60208, USA. ${ }^{3}$ Department of Analytical Chemistry, Faculty of Pharmacy, Wroclaw Medical University, 50-556 Wroclaw, Poland. ${ }^{4}$ Faculty of Chemistry, University of Wroclaw, 50-383 Wroclaw, Poland. ${ }^{5}$ Materials Science Division, Argonne National Laboratory, Lemont, Illinois 60439, USA. 'Department of Materials Science and Engineering, KAIST, Daejeon 34141, Korea

The perception of organic crystals as brittle objects is very common. There exists, however, a group of crystals showing exceptional mechanical properties, e.g., flexibility or plasticity, ${ }^{[1-4]}$ that defies this perception. This uncommon mechanical behavior is not restricted to specific types of molecules and the most important feature characterizing their crystal structures is an anisotropy of intermolecular interactions. ${ }^{[5]}$ The uneven distribution of the interactions which differ in strength in different directions of the crystal lattice allows the molecules to change their positions when an external force is applied. Although this phenomenon has been evaluated in detail, the potential applications of bendable crystals are still limited unless other desirable properties are present.

We began the investigation with a Cambridge Structural Database survey which indicated that structural features of trisubstituted haloimidazoles, bearing at least one halogen atom, might give rise to desirable electrical properties (ferroelectricity and piezoelectricity). At the same time, the presence of halogen bonds, which were reported in literature as contacts responsible for both plastic and elastic crystal bending, could give rise to unusual mechanical properties of haloimidazoles crystalline forms. From a library of 12 synthesized haloimidazoles, we chose eight compounds that were isostructural: they crystallize in a polar Ama 2 space group and self-assemble into chains via $\mathrm{N}-\mathrm{H} \cdots \mathrm{N}$ hydrogen bonds. The compounds have the unusual propensity to produce naturally distorted ("curved") crystals. By performing rigorous computational and electric permittivity investigations, we showed that the crystal tendency to curve during growth is determined, to a substantial extent, by the contributions of weak halogen bonding to the crystal packing. What is more, we found that these bonds can be distupted, in a controllable manner, by co-crystallization of different haloimidazoles, giving as a result flexible crystals. ${ }^{[6]}$

In collaboration with the Department of Materials Science and Engineering at Northwestern University and the Materials Science Division at Argonne National Laboratory, we have demonstrated that the singlecomponent crystals and the flexible mixed crystals of haloimidazoles possess piezoelectric and ferroelectric properties along the crystallographic polar axis (parallel to the crystal long axis).[6] It is conceivable that haloimidazoles may also exhibit antiferroelectric properties along the non-polar $a$-axis. Although several attempts were made to investigate this possibility, no conclusive evidence has been obtained in this regard and so the matter warrants further investigation.

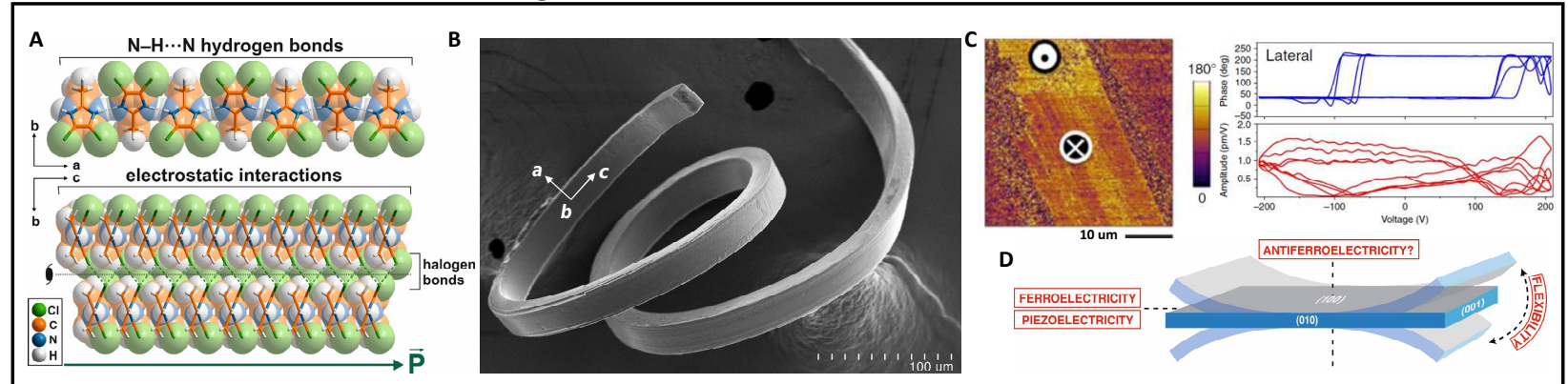

Figure 1. (A) Intermolecular interactions present in the crystal structure of investigated haloimidazoles. (B) SEM image of a naturally curved crystal of 4,5-dichloro-2-methylimidazole. (C, left) PFM image of a flexible crystal showing a domain structure. (C, right) Lateral amplitude and phase hysteresis loops obtained by a pulse dc mode. (D) Summary of crystal properties.

\section{References:}

[1] S. Ghosh \& C. M. Reddy, Angew. Chem. Int. Ed. 2012, 51, 10319-10323

[2] S. Takamizawa \& Y. Takasaki, Angew. Chem. Int. Ed. 2015, 54, 4815-4817

[3] S. Takamizawa \& Y. Miyamoto, Angew. Chem. Int. Ed. 2014, 53, 6970-6973
[4] M. K. Panda et al., Nature Chemistry 2015, 7, 65-72

[5] A. Mukherjee \& G. R. Desiraju, IUCrJ 2014, 1, 49-60

[6] M. Owczarek et al., Nat. Commun. 2016, 7, 13108 